\title{
A COMPARISON OF UAV AND TLS DATA FOR SOIL ROUGHNESS ASSESSMENT
}

\author{
M. Milenkovića,*, W. Karel ${ }^{\mathrm{a}}$, C. Ressl ${ }^{\mathrm{a}}$, N. Pfeifer ${ }^{\mathrm{a}}$, \\ ${ }^{a}$ Dept. of Geodesy and Geoinformation, Vienna University of Technology, Gußhausstraße 27-29, 1040 Vienna, Austria \\ (Milutin.Milenkovic, Wilfried.Karel, Camillo.Ressl, Norbert.Pfeifer)@geo.tuwien.ac.at
}

\section{Commission V, WG V/5}

KEY WORDS: Autocorrelation function, Correlation length, Power spectral density, Fractal dimension, Error propagation, Bundle adjustment

\begin{abstract}
:
Soil roughness represents fine-scale surface geometry which figures in many geophysical models. While static photogrammetric techniques (terrestrial images and laser scanning) have been recently proposed as a new source for deriving roughness heights, there is still need to overcome acquisition scale and viewing geometry issues. By contrast to the static techniques, images taken from unmanned aerial vehicles (UAV) can maintain near-nadir looking geometry over scales of several agricultural fields. This paper presents a pilot study on high-resolution, soil roughness reconstruction and assessment from UAV images over an agricultural plot. As a reference method, terrestrial laser scanning (TLS) was applied on a $10 \mathrm{~m}$ x $1.5 \mathrm{~m}$ subplot. The UAV images were self-calibrated and oriented within a bundle adjustment, and processed further up to a dense-matched digital surface model (DSM). The analysis of the UAV- and TLS-DSMs were performed in the spatial domain based on the surface autocorrelation function and the correlation length, and in the frequency domain based on the roughness spectrum and the surface fractal dimension (spectral slope). The TLS- and UAV-DSM differences were found to be under $\pm 1 \mathrm{~cm}$, while the UAV DSM showed a systematic pattern below this scale, which was explained by weakly tied sub-blocks of the bundle block. The results also confirmed that the existing TLS methods leads to roughness assessment up to $5 \mathrm{~mm}$ resolution. However, for our UAV data, this was not possible to achieve, though it was shown that for spatial scales of 12 $\mathrm{cm}$ and larger, both methods appear to be usable. Additionally, this paper suggests a method to propagate measurement errors to the correlation length.
\end{abstract}

\section{INTRODUCTION}

Roughness is a property of surfaces, required to understand and model interaction at these surfaces, e.g. in hydraulics, radar remote sensing, or soil erosion. The assessment of roughness has been traditionally performed by mechanical profiling (Mattia et al., 2003), but this is naturally restricted by the length of the ruler and the effort to place it at different locations. With efficient methods for acquiring point clouds at high resolution like terrestrial laser scanning (TLS) and high density image matching (Lichti and Jamtsho, 2006) (Rothermel et al., 2012) (RiekeZapp and Nearing, 2005), new possibilities for assessing roughness arise. The range envelope for which roughness should be quantified depends on the application. In radar remote sensing, but likewise in the optical domain, the backscattering behavior depends on the roughness in relation to the wavelength (Ulaby et al., 1986). As an example, Sentinel-1 has a wavelength of 5.5 $\mathrm{cm}$. Thus, the roughness between a few $\mathrm{mm}$ and up to several decimeters should be modeled.

The shape of a rough surface can be modeled as a random process, as a scalar function of the $\mathrm{X}$ - and Y-coordinate (Verhoest et al., 2008). Assessing the roughness therefore requires the study of a larger area. Using again the example of Sentinel-1, which has a resolution of $5 \mathrm{~m} \times 20 \mathrm{~m}$, a highly detailed surface model should be derived for an extent of several multiples of $20 \mathrm{~m}$.

Terrestrial laser scanning has proven to be a suitable tool for modeling the roughness at the required scales. Positioning the TLS at large height above the ground, and restricting the ranges used, guarantees that roughness content placed close to the Nyquist frequency is assessed with 5\% accuracy or better (Milenković et al.,

\footnotetext{
${ }^{*}$ Corresponding author
}

2015). However, for larger areas this procedure is less practicable due to the number of required stand points. Airborne acquisition, in contrast, allows large area coverage. However, standard photogrammetric flights cannot provide the resolution required. Lower flying heights would be necessary to reach it.

Using a UAV these low flying heights become possible: the airborne close range approach. Above that, low cost components (small UAVs, consumer cameras), make this approach interesting. However, little work has been performed on very high resolution mapping of irregular surfaces by UAVs. In (Eltner et al., 2013), UAV images with 2-4 $\mathrm{mm}$ ground sampling distance (GSD) were used to provide $\mathrm{cm}$ to sub-cm accurate DSMs for multi-temporal soil erosion monitoring. Also (Mancini et al., 2013) reported that UAV data with $6 \mathrm{~mm}$ resolution was acquired to monitor beach dune geometry. The heights reconstructed from this UAV data were within a few centimeters compared to the heights of a TLS data set used as the reference.

The aim of this article is to investigate if the quality obtained by TLS can be achieved also by light weight UAV image based acquisition. Specifically, in this paper data is acquired over a plot of bare soil, by TLS and with images from a UAV. The surface model from TLS serves as a reference and the model from the images is compared against it. This comparison is performed on the basis of roughness measures.

\section{EXPERIMENT SETUP AND DATA}

\subsection{Experiment Setup}

TLS and UAV data were collected over an agricultural field, located just beyond the city border of Vienna, Austria. The measurements were performed one after another in a single day in 

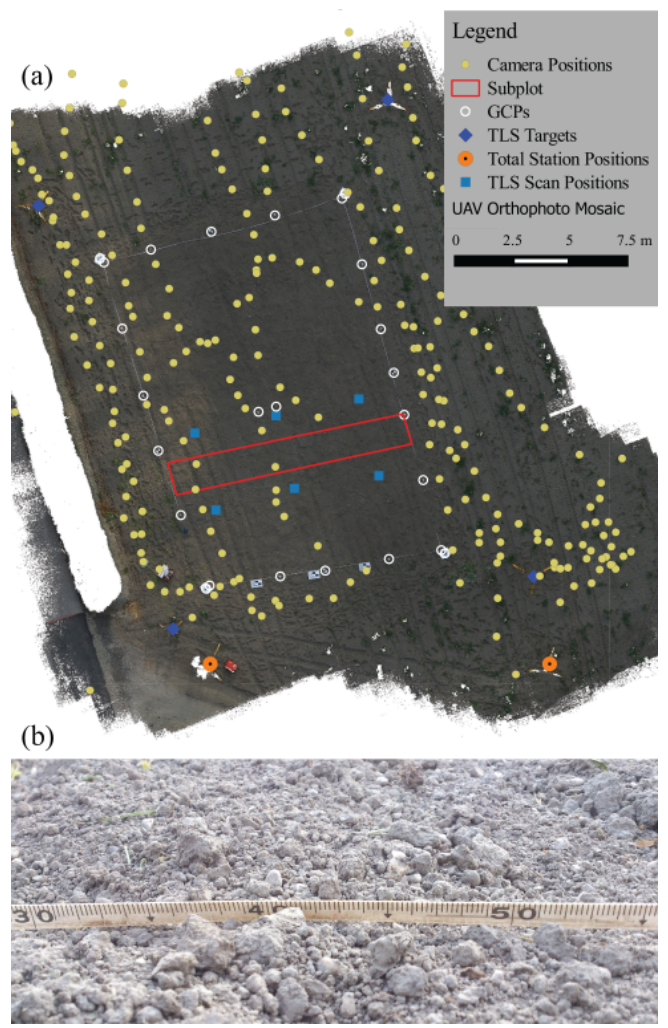

Figure 1: (a) The agricultural plot with the UAV and TLS measurement setup; (b) An image of the fine-scale roughness elements present in the plot.

June 2015. The weather was mostly sunny with some short cloud interruptions and without rain, but with wind that was strong enough to prevent the UAV from flying in autopilot mode.

The experimental setup is shown in Figure 1a, where the orthophoto derived from the UAV images was used as background. The large rectangular plot delineated by the ground control points (GCPs, the white circles in Figure 1a) was surveyed with the UAV images, while a subplot of ca. $10 \mathrm{~m} \times 1.5 \mathrm{~m}$ (the red rectangle) was surveyed with the TLS. Additionally, total station measurements were performed to define a local datum, i.e. to derive coordinates of the GCPs in the local object coordinate system. The GCPs were symmetrically distributed around the plot and separated at maximum $3 \mathrm{~m}$ from one another. This, rather small, GCP spacing was selected to minimize systematic deformations in the object space due to residual errors in the images.

The agricultural soil plot contained roughness elements over several scales. The most prominent ones were low-frequency periodic surface components ( 2 to $3 \mathrm{~cm}$ in the amplitude) which were introduced with a mechanical, seed-bed preparation tool. On top of these components, there were randomly distributed soil clods (soil aggregates up to a few centimeters), very fine soil grains (Figure 1b), and a number of small, individual vegetation patches (up to a few decimeters). Since the purpose of this study was to characterize the bare soil roughness, this vegetation was removed from the plot before data acquisition.

\subsection{UAV Images}

The images were collected with a Sony $\alpha$ ILCE-6000, an interchangeable lens camera mounted on an octocopter (Figure 4). The camera's sensor size is $23.5 \mathrm{~mm} \times 15.6 \mathrm{~mm}$, which corresponds to pixel size of $3.9 \mu \mathrm{m}$. The camera was combined with a
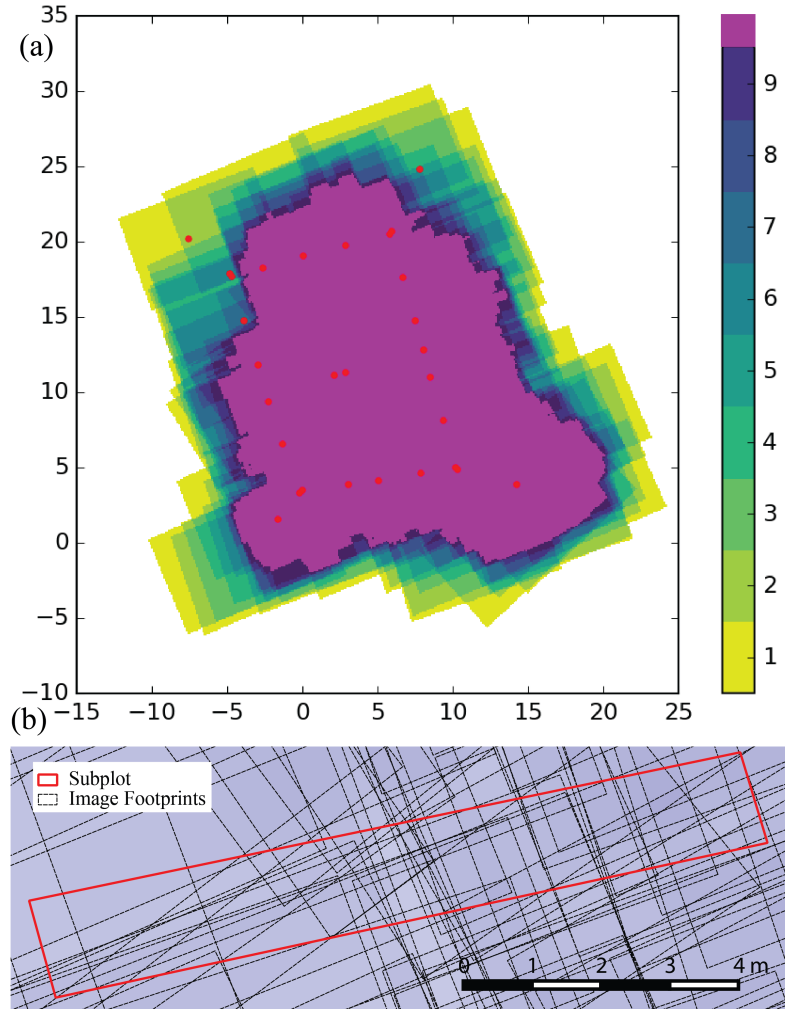

Figure 2: (a) Image density map (the GCPs are plotted as red dots); (b) Image footprints within the subplot.

zoom lens Sony AF E 16-70mm 4.0 ZA OSS, the focal length of which was $52 \mathrm{~mm}$ during image acquisition.

Due to strong wind, the flight was performed manually and as much as possible parallel to the two longer sides of the plot. The flight took 13 minutes to acquire 254 images, which were predominately distributed along the two longer sides of the plot, but with some of them also distributed along the plot's central axis (Figure 1). Thus, the acquired images were not distributed in the well-know regular strip pattern. Nevertheless, as shown in Figure $2 \mathrm{a}$, the plot was covered everywhere with at least 9 images. The average flying height was $22 \mathrm{~m}$, which resulted in a ground sampling distance (GSD) below $2 \mathrm{~mm}$. The images were selfcalibrated in a bundle block adjustment procedure.

The image resolution was then examined based on the edge spread function, also known as edge response (Perko et al., 2004)(Jacobsen, 2009). The edge spread function was calculated by the QuickMTF software (www.quickmtf.com) using the special test charts (provided by the software company) which were printed and fixed to flat plates on the ground during image acquisition (Figure 3a-b). The estimated modulation transfer functions for each color are shown in Figure 3. According to the $50 \%$ modulation threshold, the reported resolution is 6 pixels, which is approximately $12 \mathrm{~mm}$ in the object space. This resolution is notably lower compared to the images' GSD (ca. $2 \mathrm{~mm}$ ). One of the reasons for this degradation of image resolution is motion blur which is most probably caused by an inappropriate shutter time setting and vibration of the UAV.

\subsection{TLS Data}

In this experiment, the data collected with a $\mathrm{Z}+\mathrm{F}$ Imager 5010c served as reference. Generally, UAV images observe soil from above, providing better viewing geometry compared to TLS data 
(a)
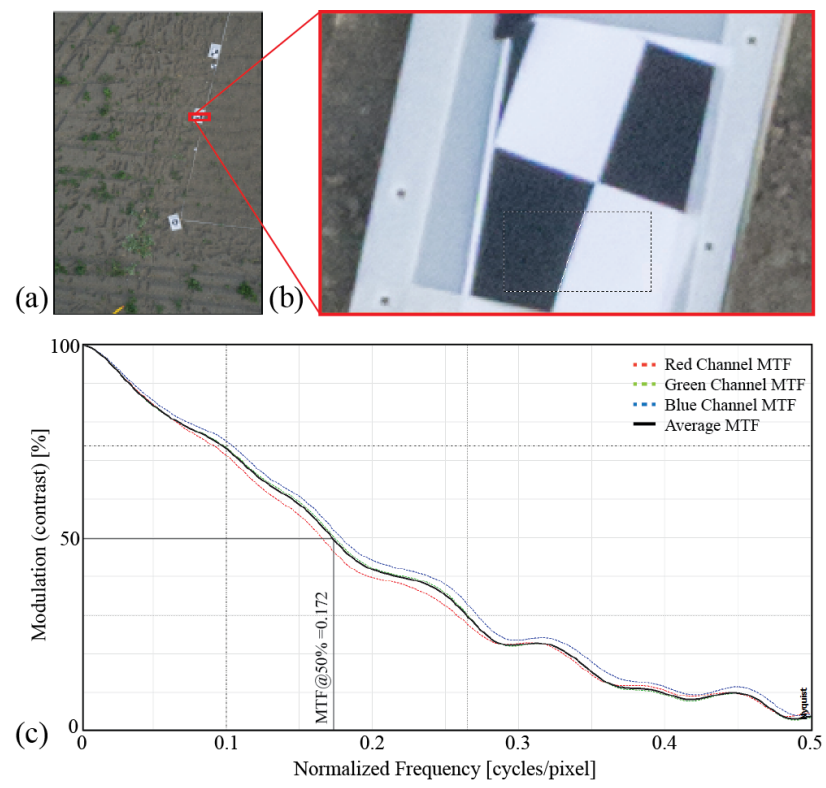

Figure 3: The modulation transfer function (MTF) corresponding to the edge spread function (measured in the in-flight direction) of an acquired image.

taken from classical geodetic tripods. However, UAV images are collected in a kinetic mode, which degrades the resolution by several pixels due to motion blur. On the other hand, the scanner itself is static and a single measurement takes less than one microsecond. Moreover, the TLS was applied here from a high tripod (instrument height $>2.5 \mathrm{~m}$ ) and with small ranges $(<6 \mathrm{~m})$, which provided more favorable viewing geometry and higher resolution compared to classical TLS setups and our UAV data.

2.3.1 Measurement Setup The subplot was scanned with 6 scan positions in total, where 3 of them were taken along each of the two longer sides of the subplot. To minimize occlusions and incidence angles, the scan positions were set approximately opposite to one another and the scanner was placed on a high tripod (Figure 4). The instrument height ranged from $2.55 \mathrm{~m}$ to $2.72 \mathrm{~m}$. Scanning was performed in the high-quality and highsampling mode, providing 10000 range measurements per full circle. The scanning started at 19:10 CET and completed in 50 minutes, taking on average 9 minutes per station.

The TLS points collected within the subplot have the following characteristics. $95 \%$ of the points have a range smaller than 4.5 $\mathrm{m}$. Since the scanner has a precise laser beam $(3 \mathrm{~mm}$ beam diameter at the exit and a divergence of $0.3 \mathrm{mrad}$ ), the diameter of the laser-beam footprint was below $5 \mathrm{~mm}$ all over the subplot. $90 \%$ of the points have an incidence angle below $52^{\circ}$, which fulfills the requirements suggested by (Milenković et al., 2015) for TLS in roughness applications. The average point density within the subplot is 85 points $/ \mathrm{m}^{2}$, while $90 \%$ of the subplot has at least 40 points $/ \mathrm{m}^{2}$, i.e. the point spacing within the subplot is $1.5 \mathrm{~mm}$ or smaller. This means that the laser-beam footprints of neighboring points were overlapping one another, which is also known as the correlated sampling mode where the resolution of the TLS data is rather limited by the laser beam footprint (Lichti and Jamtsho, 2006) and (Milenković et al., 2015). This means that the resolution of this TLS data is approximately $5 \mathrm{~mm}$.

2.3.2 TLS Data Pre-Processing The six raw TLS scans were first preprocessed in " $\mathrm{Z}+\mathrm{F}$ Laser Control"software where the mixed-pixel and single-pixel filters were applied. These filters remove points with erroneous range values happened when the laser beam of a phase-comparison laser scanner illuminates several objects distributed along the laser line of sight (Langer et al., 2000). For our surface, this mostly happened when the laser beam simultaneously illuminated the top of a soil clod and the soil surface in the background. In addition to these filters, the intensity filter was applied to remove points with an intensity smaller than $1 \%$. These points are generally less accurate because their range determination is associated with a small signal to noise ratio (Langer et al., 2000).

The parameter values used in the above filters (mixed-pixel, singlepixel and intensity) are the default values recommended by the " $\mathrm{Z}$ + F Laser Control" software. This default parameter setting was already found to be appropriate for soil-roughness preprocessing (Milenković et al., 2015).

\section{METHODS}

\subsection{UAV Data Processing}

The UAV imagery was oriented and the camera was calibrated using OrientAL (Karel et al., 2013). Missing any direct sensor orientation data, processing started with a variation of Structurefrom-Motion (SfM) (Torr and Zisserman, 2000): image feature points are detected in each image and their neighborhoods are described, after which point pairs with similar descriptors are matched between image pairs. Relative image pair orientations are computed and outlier matches discarded. For an initial image pair, object points are forward intersected and both camera orientations and object points are optimized in a bundle block adjustment. Subsequently, additional images are added to the block one after another by spatial resection and further object points are triangulated, until all images have been oriented. Aiming at the error-free and precise orientation of all images, robust methods are employed at all stages, and intermediate bundle block adjustments are executed after the addition of further images. Already the pairwise image matching takes place in Euclidean space, using an approximate interior orientation derived from Exif data. In contrast to other software packages, OrientAL estimates a variable set of lens distortion parameters, depending on their signif-

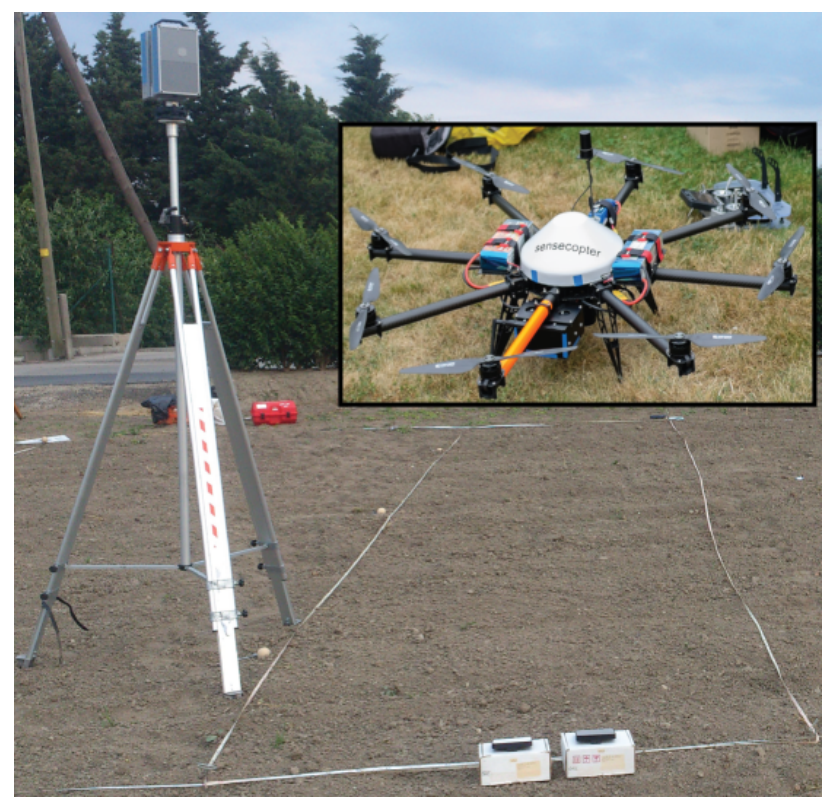

Figure 4: The TLS and UAV instruments used in this experiment. 
icance and stability, and to better handle perspective image distortion, we use Affine SIFT features (Morel and G.Yu, 2009), which are not only scale and rotation invariant, but also invariant to affine distortion. Finally, the maximum admissible image point residual norm is not a fixed parameter, but is derived from the data itself.

Using these relative image orientations, we forward intersect the GCPs into model space using their manual image observations, apply the resulting similarity transform to the bundle block, and introduce additional observations for the GCPs in image and object space, taking into account their stochastic nature. While we assumed the standard deviations of feature point image observations a priori to be 1 pix, image observations of control points were given the higher precision of 0.1 pix due to their higher definition accuracy. Observations of control point coordinates in object space were assigned a standard deviation of $1 \mathrm{~mm}$ a priori. This resulted in median residual norms for feature image points of 0.46 pix, for GCP image points of 0.97 pix, and for GCP object points of $14 \mathrm{~mm}$. The rather large residuals of GCP image points may be a result of the weak block geometry with 2 flight strips of extremely high overlap along-track, but low overlap across-track.

\subsection{Data Co-Registration}

Before interpolation and data comparison, it was necessary to bring the UAV and TLS data into the same local object coordinate system (LOCS). This was done trough several co-registration steps. First, the individual TLS scans were georeferenced to the LOCS with the help of the control points measured with the total station. This procedure was performed using an adjustment procedure implemented in " $\mathrm{Z}+\mathrm{F}$ Laser Control" software. Then, in an additional step, the individual TLS scans were co-registered to one another using a variant of the iterative closest point (ICP) algorithm implemented in the software OPALS (Glira et al., 2015). This step minimizes the point-to-surface distance among all the scans, and for our 6 scans data, the standard deviation of this distance after the ICP procedure was $0.8 \mathrm{~mm}$. This value is just two times the specified measurement noise of the $\mathrm{Z}+\mathrm{F}$ IMAGER $5010 \mathrm{c}$ scanner, which suggests at very good co-registration of the TLS scans. The six TLS scans were then merged into one TLS point-cloud block.

The UAV images were oriented trough a bundle adjustment procedure where the GCP coordinates measured with the total station were also used as observations. Thus, the resulting exterior camera parameters were already in the LOCS. This means that the densely matched points as well as the automatically derived UAV DSM are both in the LOCS. Still, in order to remove any remaining residual errors in the absolute orientation and to optimize the relative orientation between UAV DSM and TLS DSM, another ICP run was applied to both DSMs. The standard deviation of the final point-to-surface distances between UAV DSM and the TLS block was $4 \mathrm{~mm}$.

\subsection{Detrending and DSM Interpolation}

Roughness analysis is generally performed on detrended heights. Thus, the UAV and TLS data were additionally detrended here using the regression plane fitted trough the TLS points within the subplot. This means that the detrended UAV and TLS heights were calculated as the normal residuals to the regression plane, while the planar coordinates were additionally reduced to the center of gravity of the TLS points.

The TLS DSM was interpolated from the detrended TLS pointcloud block. The interpolation was done for a $1 \mathrm{~mm}$ grid spacing and with the moving plane interpolation applied to points within a $2.5 \mathrm{~mm}$ neighborhood radius. This neighborhood size was selected to match the size of the laser beam footprint within the plot. The UAV DSM was automatically produced by the SURE software (Rothermel et al., 2012), where the bundle results were supplied as input.

\subsection{Roughness Assessment}

Surface roughness is treated here in two ways: (a) through the so-called classical parametrization, i.e. as a zero-mean Gaussian process characterized by the root mean square (RMS) height, autocorrelation function (ACF) and correlation length $(l)$ (Verhoest et al., 2008), and (b) as a band-limited random fractal surface characterized by the spectral slope $(\alpha)$, i.e. fractal dimension $D=\frac{5-\alpha}{2}$ (Davidson et al., 2003). Both parametrizations were estimated as in (Milenković et al., 2015), i.e. from linearlydetrended soil-roughness profiles sampled as rows of the TLS and UAV DSMs.

For the classical parametrization, first the empirical autocorrelation function was derived, with its value for a lag $\tau_{k}$ calculated as:

$$
\hat{r}\left(\tau_{k}\right)=\frac{1}{N-k} \sum_{i=1}^{N-k} z_{i} z_{i+k}
$$

where $\tau_{k}=k \cdot \Delta x$ with $k$ being the lag increment, and $\Delta x$ being the DSM's grid size. $N$ is the number of height samples in a DSM row, while $z_{i}$ and $z_{i+k}$ are the DSM row heights at positions $i$ and $i+k$, respectively. The RMS height $s$ was estimated as $s^{2}=\hat{r}(0)$, while the correlation length $l$ was calculated as in (Davidson et al., 2003), i.e. directly interpolating the normalized autocorrelation function $\rho\left(\tau_{k}\right)=\hat{r}\left(\tau_{k}\right) / \hat{r}(0)$ :

$$
l=\tau_{m}+\left(e^{-1}-\rho\left(\tau_{m}\right)\right) \frac{\tau_{m+1}-\tau_{m}}{\rho\left(\tau_{m+1}\right)-\rho\left(\tau_{m}\right)},
$$

where $\tau_{m} \leq l \leq \tau_{m+1}$ and $\rho\left(\tau_{m}\right) \leq e^{-1} \leq \rho\left(\tau_{m+1}\right)$.

For the fractal parametrization, it was necessary first to estimate the power spectral density (the roughness spectra). This was done by calculating the assembly-average of the hamming-windowed periodograms derived from the sampled DSM rows (Milenković et al., 2015). Based on the derived roughness spectra, the spectral slope $\alpha$ is calculated as the slope of a regression line used to approximate the roughness spectrum on the logarithmic scale and within a particular frequency band (Dierking, 1999). In the linear-scale frequency domain, the latter is equivalent to:

$$
S(f)=c \cdot f^{-\alpha}
$$

where $f$ is the spatial frequency, $\log _{10}(c)$ is the intercept of the regression line, and $S(f)$ is the roughness spectrum.

3.4.1 Roughness Levels of Detail The roughness level of detail present in the TLS- and UAV-DSM is judged in two ways: (a) qualitatively, by visual comparison of the DSMs' shaded models, and (b) quantitatively, by calculating the difference of the estimated UAV and TLS roughness spectra. The roughness spectra difference can reveal the spatial wavelengths (scales) over which two corresponding DSMs share the same roughness information. According to the study of (Oh and Kay, 1998), which is based on synthetic data, the roughness spectra difference should be below $1 \mathrm{~dB}$ for accurate prediction of the microwave backscatter strength.

\subsection{Autocorrelation Function and Error Propagation}

The TLS DSM and UAV DSM which are used to derive the autocorrelation functions are not free from measurement and inter- 
(a)

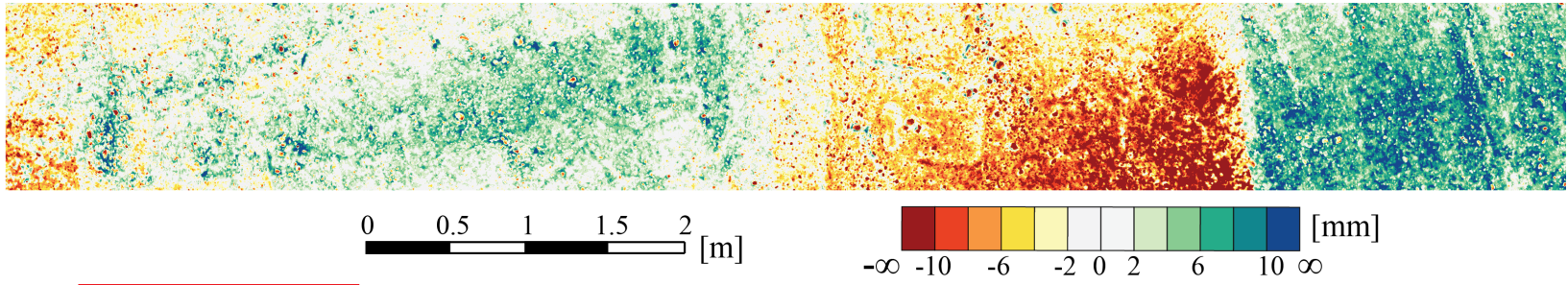

(b)

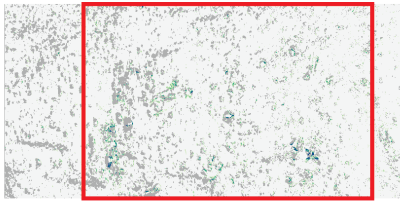

(c)

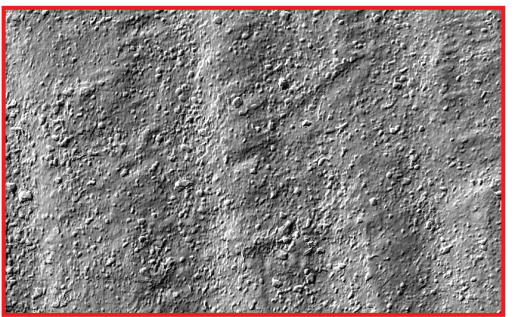

(d)
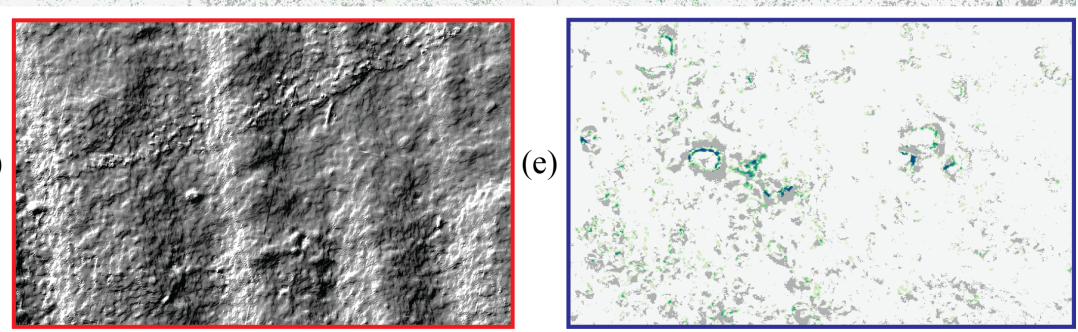

Figure 5: (a) Difference map between the TLS- and the UAV-DSM; (b) Min-max distance map derived from the DSMs of six overlapping single-TLS scans; (c) and (d) are shaded TLS- and UAV-DSM, respectively, from the region marked by the red rectangle in (b); (e) is the zoom-in to the blue rectangle in (b).

polation errors. Thus, this section considers how these errors can be quantified and propagated further to to the autocorrelation estimates.

For the TLS DSM, the height errors are quantified with the movingplane interpolation error $\sigma_{z}$. This error is derived for each grid position by propagating the RMS error of the plane adjustment procedure of the neighboring points during the interpolation process. Since the neighborhood used in our case is small $(2.5 \mathrm{~mm}$ radius), the interpolation error can be seen as a mixture of the measurement noise as well as the modeling error caused by a planar approximation of roughness elements like soil clods.

For the UAV DSM, the height error is quantified as the standard deviation $\left(\sigma_{M A D}\right)$ derived from the median of absolute differences of the dense-matched point's heights within $6 \mathrm{~mm}$ neighborhood radius. The dense-matched points are one of the outputs provided by SURE, and they are calculated while matching a base image with several neighboring images (Rothermel et al., 2012). This means that for each pixel of a base image there are several reconstructed heights corresponding to the neighboring image pairs. Therefore, the DSM heights (also provided by SURE) can be seen as a median-like filter of the matched points. The $\sigma_{M A D}$ estimation radius $(6 \mathrm{~mm})$ was set according to the image resolution in the object space (ca. $12 \mathrm{~mm}$ ).

$\sigma_{z}$ and $\sigma_{M A D}$ are available for each height of the TLS DSM and UAV DSM, respectively. Based on Eq.(1), it is possible to propagate $\sigma_{z}$ and $\sigma_{M A D}$ further to the autocorrelation values for each particular lag. This can be easily done when Eq.(1) is seen as linear combination, i.e. as a product of a row vector $\mathbf{h}_{k}^{\top}$ and a column vector $\mathbf{z}$ :

$$
\hat{r}\left(\tau_{k}\right)=\frac{1}{N-k} \sum_{i=1}^{N-k} z_{i} z_{i+k}=\mathbf{h}_{k}^{\top} \cdot \mathbf{z},
$$

where:

$$
\mathbf{h}_{k}=\frac{1}{N-k}\left[\begin{array}{c}
\mathbf{0} \\
z_{1} \\
\vdots \\
z_{N-k}
\end{array}\right] \text {, and } \mathbf{z}=\left[\begin{array}{c}
z_{1} \\
z_{2} \\
\vdots \\
z_{N}
\end{array}\right]
$$

while the first $k$ elements of $\mathbf{h}_{k}$ are zeros. For the zero-lag autocorrelation $\hat{r}(0)$, it holds: $\mathbf{h}_{0}=\frac{1}{N} \mathbf{z}$. Following the error propagation law for $\mathbf{h}_{k}$ and $\mathbf{z}$ (both contain individual random variables), the expression for the variance of a single-lag autocorrelation value $\hat{r}\left(\tau_{k}\right)$ is:

$$
\hat{\sigma}_{r_{k}}^{2}=\mathbf{h}_{k}^{\top} \cdot \boldsymbol{\Sigma}_{\mathbf{z z}} \cdot \mathbf{h}_{k}+\mathbf{z}_{k}^{\top} \cdot \boldsymbol{\Sigma}_{\mathbf{h}_{k} \mathbf{h}_{k}} \cdot \mathbf{z}_{k}
$$

$\boldsymbol{\Sigma}_{\mathbf{z z}}$ is a full diagonal matrix containing variances $\sigma_{z_{i}}^{2}$, while $\boldsymbol{\Sigma}_{\mathbf{h}_{k} \mathbf{h}_{k}}$ is the same as $\boldsymbol{\Sigma}_{\mathbf{z z}}$, but with the first k diagonal elements equal to zero. Finally, to compute the variance of $\hat{r}\left(\tau_{k}\right)$ for the TLS DSM, the $\sigma_{z_{i}}^{2}$ values are replaced with $\sigma_{z}^{2}$, while in case of the UAV DSM, the $\sigma_{M A D}^{2}$ is used instead.

\section{RESULTS AND ANALYSIS}

\subsection{Accuracy of Data Co-Registration}

Improper data co-registration may lead to false roughness analysis. Thus, two elevation-difference rasters were prepared to report on the co-registration accuracy: one to check the co-registration of the individual TLS scans, and another to check the co-registration of the TLS DSM and UAV DSM.

The co-registration of the individual TLS scans was checked with the minimum-maximum elevation difference (range) for each pixel of a set of six overlapping DSMs interpolated from the individual TLS scans. Figure 5b shows the color-coded range map for our 6 TLS scans over the subplot with a pixel size of $1 \mathrm{~mm}$. Since the range values are non-negative, the colors correspond only to the right half of the given color palette. The gray values show the 
areas which contain just one individual TLS DSM, and they occupy about $30 \%$ of the plot. The $95 \%$ of the remaining area contains range values below $2 \mathrm{~mm}$, which indicates a very good coregistration of the TLS scans. The remaining 5\% of large range values occurs mostly around the soil clod edges, which can be seen in Figure 5e. This is because the moving-plane interpolation with a neighborhood radius of $2.5 \mathrm{~mm}$ (the interpolation method used for the DSM interpolation) does not perform well on soil clod borders. However, these are generally known interpolation artifacts that can not be easily overcome with our TLS data.

Figure 5a shows the color-coded elevation differences between the UAV DSM and the TLS DSM. Due to the ICP minimization between the two DSMs performed in the preprocessing step, the differences are on average zero, and $90 \%$ of the differences are within $\pm 9 \mathrm{~mm}$. However, within this accuracy range, there are also large systematic patterns in the difference map. After careful examination, it was found that borders between regions of positive and negative differences correspond to image borders (see Figure 2b). Residual image orientation errors may be responsible for that. These orientation errors could be caused by insufficient overlap between individual image sub-blocks. Additionally, during the image acquisition, the image stabilization was switched on to reduce motion blur, which caused unstable inner geometry of the camera, and consequently, difficulties to determine a single distortion model valid for all images. With an accuracy of $\pm 9 \mathrm{~mm}$ this UAV DSM is only sub-optimal. Still, within this accuracy bound, the UAV DSM can be used for the comparison with the TLS DSM. However, this experience raises the awareness that the acquisition of the UAV images should be conducted with much more care - especially regarding the stability of the inner image geometry. For an identical image set with a stable inner geometry, the accuracy of the UAV DSM should be expected to be notably higher, and without the systematic effects shown in Figure 5a.

\subsection{Roughness Assessment in Frequency Domain}

The TLS and UAV DSMs of the whole plot are analyzed here in the frequency domain. Figure 6 shows two roughness spectrum lines derived from the TLS DSM (red) and UAV DSM (blue), respectively. They are the ensemble-average, hamming-windowed periodograms derived from 100 rows of the corresponding DSMs. To make interpretation easier, the periodograms were further smoothed with the moving average with the span of 100 elements. This procedure preserves the general trend of the periodogram while removing the undesirable variability associated with this estimator. The black line in Figure 6 shows the difference between the TLS- and UAV-based periodograms.

There are 4 frequency bands where the roughness spectra perform differently. These bands are separated with the three vertical dashed lines in Figure 6. The very right band (from the Nyquist up to the $5 \mathrm{~mm}$ wavelength) of the TLS spectrum shows a whitenoise (horizontal) roll-off, indicating that there is no further information contained. This is fully consistent with the resolution of the TLS DSM, i.e. with the diameter of the laser footprint which was up to $5 \mathrm{~mm}$ within the subplot. These frequencies are present in the periodogram because the TLS DSM was unnecessarily interpolated to $1 \mathrm{~mm}$ grid even though the resolution of the data itself was lower $(5 \mathrm{~mm})$. However, this was performed just to have an additional estimate of the resolution of our control data set, and to illustrate that the Nyquist frequency does not necessarily indicate the resolution of a DSM. Additionally, it can be seen that the roughness spectrum at these frequencies is below $20 \mathrm{~dB}$, which is several orders of magnitude smaller than the TLS measurement noise. This indicates that the measurement noise is

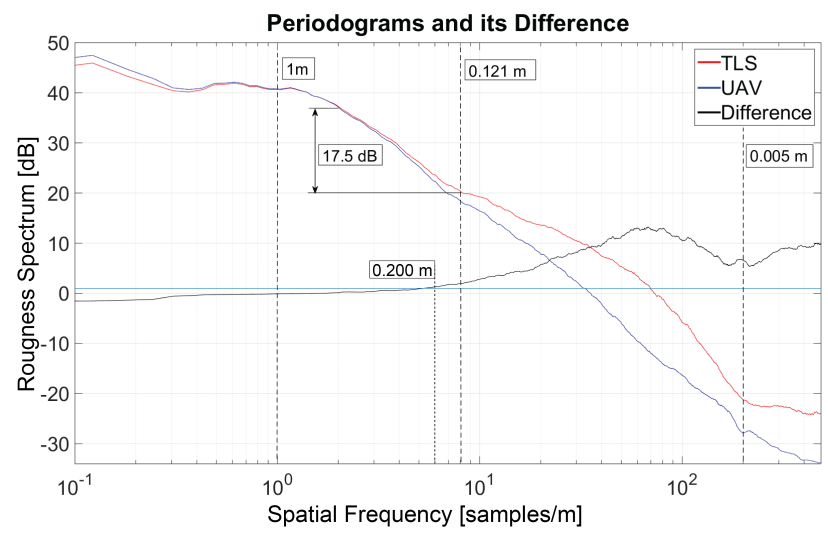

Figure 6: The PSD functions

filtered out from the TLS point cloud during the DSM interpolation. Thus, it shows that the TLS data are appropriately modeled.

From the spectra difference, it can be shown that the roughness spectra are similar in the first two frequency bands up to the $1 \mathrm{~dB}$ limit (the blue horizontal line in Figure 6). The $1 \mathrm{~dB}$ limit was selected according to a study of (Oh and Kay, 1998). This practically means that the TLS DSM can be readily replaced with the UAV DSM when the relevant roughness content is placed along wavelengths up to $20 \mathrm{~cm}$. For shorter wavelengths (from $20 \mathrm{~cm}$ till $5 \mathrm{~mm}$ ), the TLS roughness spectrum contains much more roughness information than the UAV spectrum. This can be also seen in the two shaded DSMs (Figure $5 \mathrm{c}$ and 5d), which clearly show that the TLS DSM contains much more roughness elements at these scales compared with the UAV DSM, where they are smoothed out. The latter effect is most probably a consequence of image resolution and orientation errors which directly influence the DSM.

In the second frequency band (from $1 \mathrm{~m}$ to $12 \mathrm{~cm}$ wavelengths), both spectra exhibit a linear (fractal) nature, while the power of the UAV DSM drops faster ( $17.5 \mathrm{~dB}$ per 0.5 of decade) compared with the TLS power drop ( $17.5 \mathrm{~dB}$ per 0.6 of decade). The latter is equivalent to the spectral slope values of 3.5 and 2.6 for the UAV- and TLS-DSM, respectively. Thus, in this frequency band, the UAV DSM has a slightly higher spectral slope (smaller fractal dimension) compared with the TLS DSM. This shows that even though the underlying surface is identical for both data sets, the UAV and TLS data suggest two families of surfaces with different stochastic properties. This, in turn, may cause an inconsistent prediction of the microwave backscatter energy from the same surface.

Finally, in the very left frequency band (from DC till $1 \mathrm{~m}$ wavelength), the UAV spectrum is on top of the TLS spectrum, which is opposite to its behavior along the remaining frequencies. This shows that UAV spectra has more power at these frequencies compared with the TLS DSM, which is a consequence of the systematics present in the UAV DSM (Figure 5a).

\subsection{Roughness Assessment in Spatial Domain}

The TLS and UAV DSMs of the whole plot are also analyzed in the spatial domain by comparing individual profiles and their autocorrelation functions. Figure 7 a shows single height profiles sampled at the identical location in the TLS DSM (red line) and UAV DSM (blue line). From the zoomed-in area (Figure 7b), it can be clearly seen that TLS profiles reconstructed much more roughness level of detail compared with the UAV profile. This is particularly true for the soil aggregates up to $3 \mathrm{~mm}$ in size, which 


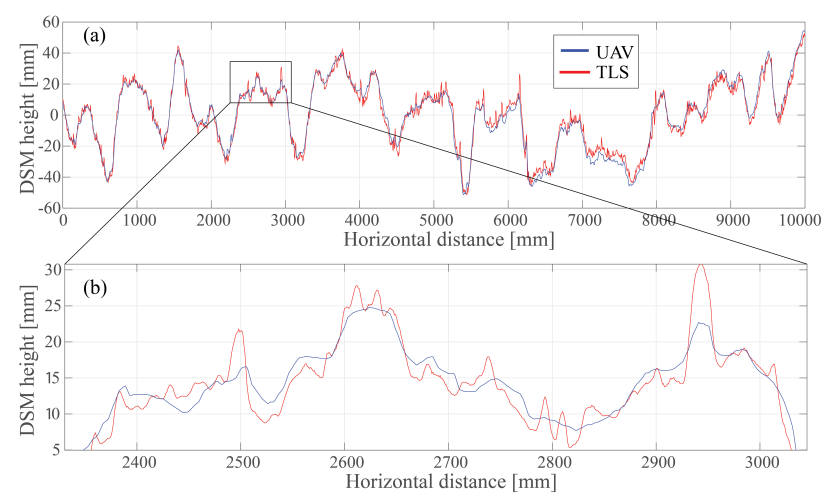

Figure 7: (a) Single TLS and UAV height profiles sampled at identical location from the corresponding DSMs; (b) Zoom-in to portions of the profiles in (a).

are completely smoothed out in the UAV profile (e.g. section from $2.6 \mathrm{~m}$ to $2.7 \mathrm{~m}$, Figure $7 \mathrm{~b}$ ). However, due to the systematic errors in the UAV DSM, this did not have an effect on the profiles' RMS height values, where the RMS height of the UAV profile $\left(s_{\text {uav }}=\right.$ $22 \mathrm{~mm})$ was found even larger than the RMS height $\left(s_{t l s}=20\right.$ $\mathrm{mm})$ of the TLS profile.

The correlation length, as a measure of roughness, was determined for both data sets. As can be seen in Figure 8 the shapes of the autocorrelation functions are similar, and the faster drop was explained as result of the finer details visible in the TLS data. The correlation length determined from the UAV images is thus approximately $15 \%$ larger than for the TLS data. In this article error propagation was used to forward the uncertainty of elevation to uncertainty of the autocorrelation function and further to the precision of the estimated correlation length. Given the lower accuracy of the image based surface model, the precision of the corresponding correlation length is poorer, too, but still only a few percent of the correlation length. This uncertainty is much lower than the offset in correlation length between the two methods. This suggests, that this error estimate is too optimistic, and a likely cause is that correlations between the individual elevation measures remained unconsidered (diagonal matrix in Eq (5)).

\section{DISCUSSION AND CONCLUSIONS}

In this experiment, a UAV data set was analyzed for soil roughness assessment. The images used in the experiment had a resolution of $12 \mathrm{~mm}$, which is low compared to the GSD of $2 \mathrm{~mm}$. The reason was the motion blur and poor lighting conditions, which led to a loss of sharpness at the individual pixel level. Terrestrial laser scanning was used as a reference method here, which does not suffer from these two effects.

Although in this experiment the TLS was selected as the reference, point clouds based on overlapping images taken very close to the object and using static acquisition can be more accurate. For example, the vertical accuracy and GSD of an image block taken $1 \mathrm{~m}$ apart from the object and with a contemporary camera and a normal-angle lens, can be a few tenths of mm (Kraus, 2007). This is notably better than the TLS co-registration accuracy and the laser footprint, both found here to be of a few $\mathrm{mm}$. Thus, in further experiments, static and very close-range overlapping imaging can be considered as the reference as well.

In Figure 5a systematic errors in the surface model derived from dense image matching became apparent, under the assumption that the TLS data serves as reference. That these systematic errors

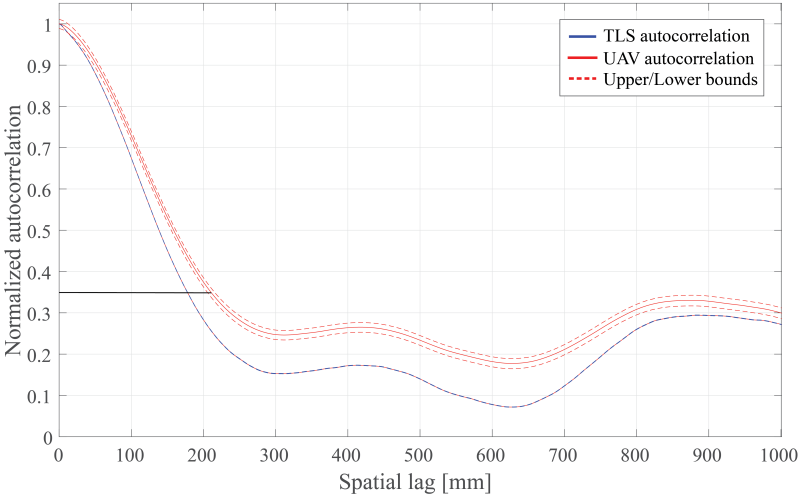

Figure 8: Normalized autocorrelation functions based on the TLS DSM and UAV DSM. The red dashed lines shows the $3 \sigma$ bounds of the propagated errors.

are related to the bundle block adjustment or the dense matching is further supported by the comparison of the pattern of Figure 5a to Figure $2 b$, which shows the overlap as grey tone and the image borders. Changes from negative to positive errors occur at regions where the overlap strongly drops. Thus, we conclude that images within the bundle formed a sub-block which has strong ties within itself, but poorer ties to neighboring sub-blocks. In this context it is noted that clods and soil grains may look differently from different perspectives, e.g. due to cast shadow, near vertical elements, etc. This would introduce high correlation between some images, which is not considered in the stochastic model of the bundle block adjustment used here.

Based on the performed analysis, one conclusion is that obtaining very high resolution images of natural bare soil surfaces with UAV remains challenging with low-cost components. The stability of the camera is of concern, which can be enhanced (turning off auto-focus, stabilizer, etc.) with suitable cameras. However, the limited stability of consumer cameras is generally known. Secondly, flying has to support image acquisition, thus avoiding motion blur due to forward motion or vibrations. Additionally, the experiment suggests that a regular block layout of the images within the block could prevent systematic errors due to forming of sub-blocks within the bundle block adjustment.

The experiment uses the methods suggested in (Milenković et al., 2015) for processing TLS data to assess surface roughness. The results are consistent with the previous study, and thus, confirm the suggested method and show that it is extendable for TLS data taken from high tripods and over a larger area. We could not prove that the low-cost UAV images considered in this experiment can deliver the same level of detail and accuracy as current TLS systems (resolution of $5 \mathrm{~mm}$ ), although improvement can be expected. However, for spatial scales of $12 \mathrm{~cm}$ and larger (Figure 6 ), both methods appear to be usable.

The experiment is also complementing the study of (Eltner et al., 2013). Both studies contribute to the same aim, i.e. developing methods for very high resolution modeling of terrain surfaces. The results of both studies are generally similar, though the GCP accuracy achieved in (Eltner et al., 2013) is apparently better compared to our results, and no systematic errors were reported there. The systematic errors in our experiments originate in (relatively) weakly tied sub-blocks of the bundle block. On the other hand, the TLS reference of this article has a co-registration error below $2 \mathrm{~mm}$, and together with the data resolution of $5 \mathrm{~mm}$, this makes it well usable as reference data. Further experimentation is necessary to build a more comprehensive understanding of the strengths and weaknesses of different approaches, and how to 
use them in synergy.

\section{ACKNOWLEDGEMENTS}

The research leading to these results has received funding from the European Community's Seventh Framework Programme ([ FP7 / 2007 - 2013 ]) under grant agreement no 606971.

We would also like to thank Melanie Rašković for helping with measurements of the ground control points as well as Peter Dorninger and Clemens Nothegger from 4D-IT GmbH for collecting the UAV images.

\section{REFERENCES}

Davidson, M., Mattia, F., Satalino, G., Verhoest, N., Le Toan, T., Borgeaud, M., Louis, J. and Attema, E., 2003. Joint statistical properties of rms height and correlation length derived from multisite 1-m roughness measurements. Geoscience and Remote Sensing, IEEE Transactions on 41(7), pp. 1651-1658.

Dierking, W., 1999. Quantitative roughness characterization of geological surfaces and implications for radar signature analysis. Geoscience and Remote Sensing, IEEE Transactions on 37(5), pp. 2397-2412.

Eltner, A., Mulsow, C. and Maas, H.-G., 2013. Quantitative measurement of soil erosion from tls and uav data. ISPRS - International Archives of the Photogrammetry, Remote Sensing and Spatial Information Sciences XL-1/W2, pp. 119-124.

Glira, P., Pfeifer, N., Briese, C. and Ressl, C., 2015. A correspondence framework for als strip adjustments based on variants of the icp algorithm. Photogrammetrie - Fernerkundung - Geoinformation 2015(4), pp. 275-289.

Jacobsen, K., 2009. Effective resolution of digital frame images. In: International Archives of the Photogrammetry, Remote Sensing and Spatial Information Sciences.

Karel, W., Doneus, M., Verhoeven, G., Briese, C., Ressl, C. and Pfeifer, N., 2013. Oriental - automatic geo-referencing and orthorectification of archeological aerial photographs. In: ISPRS Annals of the Photogrammetry, Remote Sensing and Spatial Information Sciences, II-5/W1.

Kraus, K., 2007. Photogrammetry - Geometry from Images and Laser Scans. 2 edn, De Gruyter.

Langer, D., Mettenleiter, M., Härtl, F. and Fröhlich, C., 2000. Imaging ladar for 3-d surveying and cad modeling of real-world environments. The International Journal of Robotics Research 19(11), pp. 1075-1088.

Lichti, D. D. and Jamtsho, S., 2006. Angular resolution of terrestrial laser scanners. The Photogrammetric Record 21(114), pp. 141-160.

Mancini, F., Dubbini, M., Gattelli, M., Stecchi, F., Fabbri, S. and Gabbianelli, G., 2013. Using unmanned aerial vehicles (uav) for high-resolution reconstruction of topography: The structure from motion approach on coastal environments. Remote Sensing 5(12), pp. 6880.

Mattia, F., Davidson, M., Le Toan, T., D'Haese, C., Verhoest, N., Gatti, A. and Borgeaud, M., 2003. A comparison between soil roughness statistics used in surface scattering models derived from mechanical and laser profilers. Geoscience and Remote Sensing, IEEE Transactions on 41(7), pp. 1659-1671.
Milenković, M., Pfeifer, N. and Glira, P., 2015. Applying terrestrial laser scanning for soil surface roughness assessment. Remote Sensing 7(2), pp. 2007.

Morel, J. and G.Yu, 2009. ASIFT: A new framework for fully affine invariant image comparison. SIAM Journal on Imaging Sciences.

Oh, Y. and Kay, Y. C., 1998. Condition for precise measurement of soil surface roughness. Geoscience and Remote Sensing, IEEE Transactions on 36(2), pp. 691-695.

Perko, R., Klaus, A. and Gruber, M., 2004. Quality comparison of digital and film-based images for photogrammetric purposes. In: International Archives of Photogrammetry, Remote Sensing and Spatial Information Sciences.

Rieke-Zapp, D. H. and Nearing, M. A., 2005. Digital close range photogrammetry for measurement of soil erosion. The Photogrammetric Record 20(109), pp. 69-87.

Rothermel, M., Wenzel, K., Fritsch, D. and Haala, N., 2012. SURE: Photogrammetric surface reconstruction from imagery. In: Proceedings LC3D Workshop, Berlin, December 2012.

Torr, P. H. and Zisserman, A., 2000. Feature based methods for structure and motion estimation. In: Vision Algorithms: Theory and Practice, Springer, pp. 278-294.

Ulaby, F., Moore, R. and Fung, A., 1986. Microwave Remote Sensing: Active and Passive. Artech House microwave library, Addison-Wesley Publishing Company, Advanced Book Program/World Science Division.

Verhoest, N. E., Lievens, H., Wagner, W., Álvarez-Mozos, J., Moran, M. S. and Mattia, F., 2008. On the soil roughness parameterization problem in soil moisture retrieval of bare surfaces from synthetic aperture radar. Sensors 8(7), pp. 4213-4248. 